\title{
Cinémas
}

Revue d'études cinématographiques

Journal of Film Studies

\section{CARCAUD-MACAIRE, Monique et Jeanne-Marie CLERC. Pour une lecture sociocritique de l'adaptation cinématographique. Propositions méthodologiques. Préface de Edmond Cros. Montpellier : L'Institut de sociocritique (ISM), Collection " Études sociocritiques ", 1995, 278 p.}

\section{Jacqueline Viswanathan}

Volume 7, numéro 1-2, automne 1996

URI : https://id.erudit.org/iderudit/1000942ar

DOI : https://doi.org/10.7202/1000942ar

Aller au sommaire du numéro

Éditeur(s)

Cinémas

ISSN

1181-6945 (imprimé)

1705-6500 (numérique)

Découvrir la revue

Citer ce compte rendu

Viswanathan, J. (1996). Compte rendu de [CARCAUD-MACAIRE, Monique et Jeanne-Marie CLERC. Pour une lecture sociocritique de l'adaptation cinématographique. Propositions méthodologiques. Préface de Edmond Cros. Montpellier : L'Institut de sociocritique (ISM), Collection « Études sociocritiques », 1995, 278 p.] Cinémas, 7(1-2), 239-244.

https://doi.org/10.7202/1000942ar d'utilisation que vous pouvez consulter en ligne. 
CARCAUD-MACAIRE, Monique et Jeanne-Marie CLERC. Pour une lecture sociocritique de l'adaptation cinématographique. Propositions méthodologiques. Préface de Edmond Cros. Montpellier: L'Institut de sociocritique (ISM), Collection "Études sociocritiques", 1995, $278 \mathrm{p}$.

Depuis le livre séminal de George Bluestone en 1957, les études de l'adaptation cinématographique se sont multipliées, surtout dans le contexte anglo-saxon. Une bibliographie récente en compte plus de 100. Les études générales, dans la foulée de Bluestone, ont tenté de saisir le processus de transcodage d'un média à l'autre, mais ont aussi souvent adopté un point de vue évaluatif et même parfois normatif, jugeant de l'échec ou de la réussite d'une adaptation en fonction de sa fidélité à l'original. Une telle analyse constate presque toujours l'appauvrissement du contenu du roman et condamne le film. Cette impasse a mené plusieurs critiques à conclure qu'on ne pouvait juger un film, même adapté, que comme une œuvre à part entière. Plus récentes sont les nombreuses études spécialisées qui se consacrent à l'adaptation dans le contexte d'une littérature ou d'un cinéma national (The American Novel and Film; the English Novel and Film...) ou un écrivain (Shakespeare, Steinbeck, Faulkner, Graham Greene... on Film). Ces analyses ont souvent un grand intérêt particulier, mais contribuent peu à la théorie et méthodologie de la critique de l'adaptation en général. Le livre de Monique Carcaud-Macaire et JeanneMarie Clerc constitue une contribution importante dans ce domaine. De plus, il se concentre sur une approche sociocritique, un aspect qui n'avait été que très peu développé auparavant, à l'exception de l'étude de Patrick Cattrysse' qui élargit, elle aussi, l'approche comparative roman/film pour faire intervenir d'autres systèmes modélisants liés au contexte social. 
Par ailleurs, Pour une lecture sociocritique de l'adaptation cinématographique. Propositions méthodologiques se distingue des études précédentes en se rattachant à la théorie/méthodologie générale de la création artistique au sein d'une société, théorie développée par le centre de recherches de l'université de Montpellier, dirigé par Edmond Cros. Pour bien comprendre l'argumentation et la méthode de Monique Carcaud-Macaire et Jeanne-Marie Clerc, il importe de saisir la démarche particulière de cette sociocritique. Celle-ci s'attache avant tout à expliquer les phénomènes de transfert de forme à forme, de média en média. Elle se rapproche davantage d'une anthropologie structurale de l'imaginaire que de l'histoire ou de la sociologie. Il s'agit de comprendre et d'expliquer le passage du génotexte (réservoir de l'imaginaire d'un groupe social: mythes, symboles, images, représentations...) au phénotexte, c'est à dire l'œuvre particulière où se concrétise cet imaginaire. D'autre part, tout phénotexte est le produit de la médiation dynamique d'un modèle formel, lié lui aussi au contexte social. Chaque analyse de ce volume applique un aspect du modèle exposé dans l'introduction. L'ouvrage comporte donc ainsi une grande cohérence théorique et méthodologique.

Pour une lecture sociocritique de l'adaptation cinématographique part du principe que toute adaptation est en premier lieu une lecture menant à une écriture, suivant un processus de déconstruction (du texte de départ) et de reconstruction (scénario/film), en fonction de deux génotextes différents. Suivant cette optique, l'adaptation se présente comme un phénomène privilégié pour l'analyse sociocritique de la médiation entre deux textes. L'adaptation est donc envisagée moins comme l'expression d'une créativité consciente et individuelle que comme une transformation due à la modélisation du média et à la translation d'un génotexte différent. L'originalité singulière de cette approche consiste à explorer les écarts entre texte de départ et adaptation comme autant d'indices révélateurs de la médiation. Ainsi se différencie-t-elle des nombreuses analyses d'adaptations cinématographiques qui caractérisent les écarts avec l'œuvre littéraire comme autant de trahisons ou de lacunes par rapport à l'original. Monique Carcaud-Macaire et Jeanne-Marie Clerc 
pratiquent donc une sorte d'herméneutique, découvrant, à la fois dans l'œuvre littéraire et dans son adaptation, des "latences" qui n'apparaissent qu'à travers cette confrontation. Elles identifient aussi les "interdiscours" qui ont régi la production (symboles, mythes, discours sociaux, artistiques, intertextes divers). Tout cet ensemble (génotexte), indispensable pour une analyse sociocritique, est manifestement très complexe et, puisqu'il ne se concrétise que dans des œuvres particulières, insaisissable dans son ensemble. Etant donné la portée et l'objet de cette critique, on ne peut qu'admirer la clarté et la précision des analyses.

Le premier chapitre présente un exposé historique des différentes conceptions de l'adaptation filmique de textes littéraires en France, des débuts du cinéma à la fin des années soixante. Ce survol révèle la diversité des points de vue successifs sur les rapports entre littérature et cinéma, idées souvent préconçues qui président aussi bien au choix des œuvres littéraires qu'à l'écriture du scénario: fascination pour l'image des débuts quand il s'agissait de faire visuel à tout prix, ensuite, pour les grands romans naturalistes, écueils des adaptations simplistes qui confondaient visualité littéraire et cinématographique. Une analyse détaillée du scénario d'Aurenche et Bost, adapté de La Symphonie pastorale de Gide, fait aussi ressortir tout l'écart entre la conception de départ des scénaristes, qui cherchaient à traduire la vision propre à un grand écrivain, et le script où les nombreuses différences par rapport au roman renvoient au contraire à un respect des canons éprouvés du drame bourgeois et aux exigences du cinéma de cette époque.

Après ce préambule théorique, méthodologique et historique, la majeure partie de Pour une lecture sociocritique de l'adaptation cinématographique se compose d'analyses d'œuvres spécifiques: deux versions filmées (l'une française, l'autre états-unienne) des Croix de bois de Roland Dorgelès; l'adaptation du roman d'Anne Hébert, Kamouraska; celle de Mort à Venise de Thomas Mann par Visconti et enfin, Le Carrosse d'or de Renoir, adapté de la pièce Le Carrosse du Saint Sacrement de Mérimée. Cette sélection rassemble des textes et des réalisateurs très divers. Audelà de l'avantage que comporte une telle variété pour mieux mettre à l'épreuve la méthodologie, on aurait aimé connaître les 
raisons qui ont inspiré ce choix particulier. Par ailleurs, les rapports entre ces œuvres littéraires et ces films, du point de vue de leurs "génotextes" respectifs, présentent des écarts historiques et culturels très variables. Ainsi les deux Kamouraska sont très proches mais les deux Carrosse beaucoup plus distants. Quelle est l'importance de cette distance, comment situer les analyses les unes par rapport aux autres? Je me suis posé ces questions.

Chaque chapitre procède de façon très claire et systématique: comparaison au niveau du récit (structures narratives et actantielles, redistribution des rôles), de la "microsémiotique " (modification dans les représentations déjà structurées par la langue et les images sur lesquelles travaille la création culturelle) et enfin, pratiques d'écriture fortement influencées par la spécificité créatrice du média lui-même. L'examen du roman et la comparaison entre les deux versions filmées des Croix de bois, la première française par Raymond Bernard (1931), la deuxième étatsunienne, The Road to Glory par Howard Hawks (1936), fait ressortir des écarts significatifs dans la transposition de l'espace et du temps et la distribution des rôles actoriels. Monique Carcaud-Macaire et Jeanne-Marie Clerc montrent que la version française actualise, par des images, le mythe de la victime expiatoire déjà inscrit au cœur du roman. En revanche, Howard Hawks, dans la version états-unienne, met en relief une microsémiotique du chef et du héros qui transforme profondément la signification du sacrifice; le film états-unien accentue aussi une vision manichéiste, conforme, selon les auteures, à la notion d'élection divine de la doctrine puritaine. Cette dernière serait une composante du génotexte états-unien qui aurait ainsi significativement déplacé le texte français. La corrélation entre la glorification manifeste des officiers dans Road to Glory et l'idéologie puritaine états-unienne n'est évidemment pas démontrable. Une certaine image française de la culture états-unienne peut-elle avoir influencé cette interprétation qui differe assez radicalement d'autres critiques des films de Hawks?

Les deux Kamouraska (film et roman) présentent un rapport tout différent puisqu'ils semblent, de prime abord, appartenir au même contexte socioculturel. Cependant, l'analyse démontre que "le film retourne à son insu le contenu subversif du 
roman", affaiblissant ainsi toute la thématique du défi, au niveau du contenu et de l'écriture. Ainsi, le film opte pour le "réalisme le plus classique" et par là même, le "plus banal" (p. 132). Cette observation est l'aboutissement d'une analyse extrêmement fouillée et précise, du point de vue des procédés filmiques et du contenu de séquences spécifiques du film.

La richesse de la déconstruction-reconstruction opérée par Visconti dans son adaptation de Mort à Venise de Thomas Mann révèle l'œuvre d'un grand metteur en scène. Elle permet aussi aux auteures de cette étude de donner toute leur mesure et de montrer le processus de métamorphose effectué par le tiers interprétant. En effet, Visconti actualise et développe des mythes et des symboles qui ne sont que latents dans La Mort à Venise de Mann. Le film donne à l'expérience faustienne, omniprésente dans un roman tardif de Mann, $D^{r}$ Faustus, une signification et une importance qu'il n'a pas dans la nouvelle et propose ainsi une relecture de Mann-jeune à la lumière de Mann-vieux. Le décalage culturel et chronologique est ici source d'enrichissement.

Par ailleurs, le recensement des écarts qui distinguent Le Carrosse d'or de Renoir de la pièce de Mérimée permet de mettre au jour l'apport spécifique du média cinématographique à la construction du sens. L'écriture filmique de Renoir pose constamment la question de la disparition des limites entre réalité et illusion et se rapproche ainsi davantage du théâtre baroque que du théâtre romantique de Mérimée qui donne une autre image plus stable de la réalité.

Dans ces deux derniers chapitres surtout, l'analyse minutieuse et détaillée, la rigueur méthodologique aboutissent à une vision nouvelle et enrichissante du texte littéraire et du film. Forme et signification sont appréhendées dans un même mouvement. Les concepts et les méthodes de la narratologie se combinent harmonieusement avec une sociocritique de l'imaginaire. Même si les auteures ne portent jamais explicitement un jugement de valeur, il est clair que les deux premières adaptations (Croix de bois et Kamouraska) sont décevantes alors que l'admiration des critiques pour les deux dernières se profile derrière de nombreux commentaires. Cependant, il faut préciser qu'il n'est plus question de fidélité ou de trahison, mais d'enrichissement et de 
créativité ou d'appauvrissement et de banalisation. Road to Glory de Hawks renverrait à une mythologie hollywoodienne stéréotypée alors que Kamouraska (le film) souscrit à un réalisme conventionnel. En revanche, dans Mort à Venise et Le Carrosse d'or, l'analyse met en relief la recherche originale et le génie de deux grands réalisateurs.

Au-delà de la cohérence théorique et méthodologique qui caractérise cette étude, ceux qui connaissent les ouvrages précédents ${ }^{2}$ de Jeanne-Marie Clerc pourront aussi y reconnaître la continuité d'une certaine conception du cinéma et de ses rapports à la littérature, conception caractérisée par son attention à la spécificité du média filmique qui véhicule son propre champ thématique. À travers plusieurs ouvrages, Jeanne-Marie Clerc montre combien le cinéma, média de l'apparence et de l'illusion, a révolutionné l'imaginaire contemporain. Ainsi se poursuit une réflexion à la fois rigoureuse dans sa méthode, minutieuse dans ses analyses et d'une grande profondeur au sujet de l'adaptation en particulier et des rapports entre cinéma et littérature en général.

Jacqueline Viswanathan

Université Simon Fraser

\section{NOTES}

1 Patrick Cattrysse, Pour une théorie de l'adaptation filmique. Le film noir américain. Bern : Peter Lang, 1992, et "Descriptive and Normative Norms in Film Adaptation: the Hays Office and the American Film Noir ", Cinémas, vol. 6, n" 2-3, p. 167 187.

2 Jeanne-Marie Clerc, Écrivains et cinéma, Paris: Klincksieck, 1985, et Littérature et cinéma, Paris: Nathan, 1993. 